\title{
EMERGENCY RESPONSE PLANE OF TOXIC GAS RELEASES WITH CONSIDERING VENTILATION RATIO AND METEOROLOGICAL CONDITIONS
}

\author{
YOUNG-DO JO \& KI-DONG PARK \\ Institute of Gas Safety Technology, Korea Gas Safety Corporation, South Korea.
}

\begin{abstract}
A major accident of high toxic gas, leaking of 20 tons of hydrofluoric acid [HF], was happened on 27 September 2012 in Gumi, about 200 km from Seoul, South Korea. The accident killed five workers at the site and severely injured at least 18 others, including workers and emergency personnel. The initial government response to the accident, including possible mistakes by firefighters and a sluggish evacuation of nearby residents, was sharply criticized in the Korean media. The number of industries using toxic gas also has significantly increased most of them located near inhabited areas. These facts are a huge challenge for administrations who must minimize risks around toxic gas facilities where major accidents can occur, and must provide a safe community emergency response. When toxic gas is released during for a certain time, the resident downwind area should be evacuated or stayed in a building with sealing doors and windows, and wait until the toxic gas puff has gone. The criterion for evacuating or not of residence in the building is not setup until now, which might depend on the building and meteorological conditions. In this work, we analysis the toxic gas concentration in the building with toxic gas atmospheric dispersion to help that the local communities are likely to be advised to go indoors and to close windows and doors until given further advice by the emergency manager. Wind speed and ventilation ratio of building influence highly on the concentration of toxic gas in the building. Keywords: toxic gas, indoor concentration, emergency response tree, critical distance, ventilation ratio, gas dispersion
\end{abstract}

\section{INTRODUCTION}

A major accident of high toxic gas, leaking of 20 tons of hydrofluoric acid [HF], was happened on 27 September 2012 in Gumi, about 200 km from Seoul, South Korea [1]. The accident killed five workers at the site and severely injured at least 18 others, including workers and emergency personnel. Around 12,200 people in villages and farms downwind of the accident inhaled fumes and required emergency treatment for health complaints, including nausea, chest pain, rashes and sore eyes. The accident also contaminated 212 ha crops and sickened 4,015 live stocks, and affected on 81 other firms in the area. The damage is estimated at more than $\$ 30$ million. This damage was increased due to the improper emergency response of HF leaking. The initial government response to the accident, including possible mistakes by firefighters, and a sluggish evacuation of nearby residents, was sharply criticized in the Korean media. The number of industries using toxic gas has significantly increased, most of them located near inhabited areas. This fact is a huge challenge for administrations who must minimize risks around toxic gas facilities where major accidents can occur, and must provide a safe community emergency response. 
If a community is being exposed to harm as a result of a major incident, appropriate mitigating measures must be implemented without delay. When toxic gas is released during for a certain time, toxic gas clouds may be dispersed with wind direction. In this event, people in the downwind area should be evacuated or stayed in a building with sealing doors and windows and wait until the toxic gas puff has gone. The building acts as a barrier that slows down the toxic gas entrance and the inside concentration of toxic gas would be lower than outside, as well as the toxic load to which people are exposed. The criterion for evacuating or not of residence in the building is not setup, which might depend on the building and meteorological conditions.

In this work, we analysis the toxic gas concentration in the building with toxic gas atmospheric dispersion to help that the local communities are likely to be advised to go indoors and to close windows and doors until given further advice by the emergency manager. Wind speed and ventilation ratio of building influence highly on the concentration of toxic gas in the building

\section{MATHEMATICAL MODELLING}

For a given external concentration $\left(C_{0}\right)$, the rate of change of the inside concentration of toxic gas in a confined room can be estimated by mass balance with the assumption of homogeneous concentration and no adsorption in the room [2].

$$
\frac{d C_{i}}{d t}=\dot{\eta}\left(C_{0}-C_{i}\right)
$$

Where $\bar{\eta}$ is ventilation ratio, which usually lies from $0.2 / \mathrm{hr}$ (for tightly constructed housing) to $3 / \mathrm{hr}$ (for loosely constructed housing with a median of about $0.5 / \mathrm{hr}$ ) [3]. C is the toxic gas concentration of outside and $\mathrm{C}$ is the initial concentration inside of the confined room.

For the case of puff with an instantaneous point source at ground level, coordinates fixed at the release point, constant wind only in the $\mathrm{x}$ direction with constant wind velocity; the external concentration of toxic gas at a point of ground level and the center of the moving cloud following wind direction is given by [4].

$$
C_{0}(x, y=0, z=0, t)=\frac{Q}{(2 \pi)^{3 / 2} \sigma_{x} \sigma_{y} \sigma_{z}} \exp \left[-\frac{\left(x-U_{w} t\right)^{2}}{2 \sigma_{x}^{2}}\right]
$$

Where $\mathrm{Q}$ is the total mass of released toxic gas, $\sigma$ is dispersion coefficients, $U_{W}$ is wind velocity, $\mathrm{x}$ is the downwind distance from release source, and $\mathrm{t}$ is time.

The eqns (1) and (2) will be simplified by a simple transformation coordinates. If the puff moves with the wind along the $\mathrm{x}$ axis, the solution is found by replacing the existing coordinate $\mathrm{x}$ by a new coordinate system, $\sigma=x-U_{W}$ t, that moves with the wind velocity. By defining a new variable as the difference between external and internal toxic gas concentration, $G=C_{0}-C_{\mathrm{i}}$, the eqns (1) and (2) can be rewritten as

$$
\begin{gathered}
\frac{d G}{d \tau}=\frac{d C_{0}}{d \tau}+\dot{\eta} G / U_{w} \\
\frac{d C_{0}}{d \tau}=\frac{-Q \tau}{(2 \pi)^{3 / 2} \sigma_{x}^{3} \sigma_{y} \sigma_{z}} \exp \left[-\frac{\tau^{2}}{2 \sigma_{x}^{2}}\right]
\end{gathered}
$$


Removing the rate of change of the external concentration of toxic gas by using eqns (4) and (3)

$$
\frac{d G}{d \tau}=\frac{-Q \tau}{(2 \pi)^{3 / 2} \sigma_{x}^{3} \sigma_{y} \sigma_{z}} \exp \left[-\frac{\tau^{2}}{2 \sigma_{x}^{2}}\right]+\dot{\eta} G / U_{w}
$$

Integrating the above equation to a certain time with initial condition, $\mathrm{t}=0 \Rightarrow C_{i}=0$, to obtain the inside concentration of toxic gas in a confined room.

$$
\begin{aligned}
& \text { If } \frac{1}{\sqrt{2} \sigma_{x}} \tau+\left(\frac{\sigma_{x} \dot{\eta}}{\sqrt{2} U_{w}}\right) \geq 0 \\
& C_{i}=\frac{Q}{(2 \pi)^{3 / 2} \sigma_{x} \sigma_{y} \sigma_{z}}\left[e^{\left(\dot{\eta} / U_{w}\right) \tau} \frac{\sqrt{\pi} \sigma_{x} \dot{\eta}}{\sqrt{2} U_{w}} e^{\left(\frac{\sigma_{x} \dot{\eta}}{\sqrt{2} U_{w}}\right)^{2}} \operatorname{erfc}\left(\frac{1}{\sqrt{2} \sigma_{x}} \tau+\left(\frac{\sigma_{x} \dot{\eta}}{\sqrt{2} U_{w}}\right)\right)+\text { Const }\right] \\
& \text { If } \frac{1}{\sqrt{2} \sigma_{x}} \tau+\left(\frac{\sigma_{x} \dot{\eta}}{\sqrt{2} U_{w}}\right)<0
\end{aligned}
$$

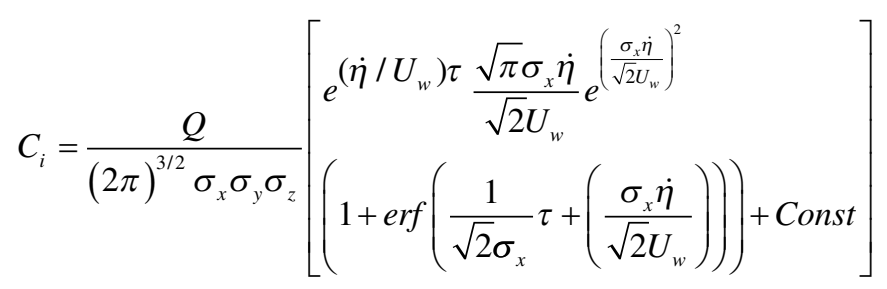

Where $\operatorname{erf}(x)$ and $\operatorname{er} f c(x)$ are error functions.

$$
\begin{gathered}
\text { Const }=-\operatorname{erfc}\left(\frac{1}{\sqrt{2} \sigma_{x}} x+\left(\frac{\sigma_{x} \dot{\eta}}{\sqrt{2} U_{w}}\right)\right) \\
\operatorname{erf}(x)=\frac{2}{\sqrt{\pi}} \int_{0}^{x} e^{-t^{2}} d t \\
\operatorname{erfc}(x)=\frac{2}{\sqrt{\pi}} \int_{x}^{\infty} e^{-t^{2}} d t
\end{gathered}
$$

If the term, $\frac{1}{\sqrt{2} \sigma_{x}} \tau+\left(\frac{\sigma_{x} \dot{\eta}}{\sqrt{2} U_{w}}\right)$, is greater than 0 , the external concentration of toxic gas is higher than the internal concentration. 


\section{ANALYSIS AND DISCUSSIONS}

A catastrophic rupture of a pipeline or tank, or a spill from a tank, such as Gumi accident, can produce a release that lasts a few seconds to a few minutes. This results in a puff-type release. For a toxic gas puff with an instantaneous point source at ground level, the gas concentration outside and inside of the building can be estimated by using eqns (2), (6) and (7). A typical changing of the toxic gas concentration inside and outside of the building shows in Fig. 1. The toxic gas concentration inside of building increases with time until it being same as the toxic gas concentration of outside, and then decreases slowly.

The ratio of the inside concentration to the outside concentration of toxic gas depends on the wind velocity and ventilation rate (other word infiltration rate). The ratio decreases with decreasing ventilation rate and increasing wind velocity as shown in Fig. 2. The curves in Fig. 2 will be on a line, if the concentration ratio is plotted against the ratio of infiltration rate per wind velocity, as shown in the Fig. 3. Generally, recent building is tightly constructed to keep warm during the winter season and infiltration rate is less than 0.3 . The inside toxic gas concentration is approximately less than two orders of external toxic gas concentration as shown in Fig. 2. The ratio of the inside concentration to the outside concentration of toxic gas depends on the dispersion coefficient as shown in the Fig. 4.

The external toxic gas concentration along to down wind direction can be estimated conservatively using Pasquill-Gifford Dispersion coefficients with stability class F, as an example.

The dispersion coefficients for the above case is given as the following [4].

$$
\sigma_{x}=\sigma_{y}=0.02 x^{0.89} \text { and } \sigma_{z}=0.05 x^{0.61}
$$

Where $\mathrm{x}$ is the distance in $\mathrm{m}$ from the release point.

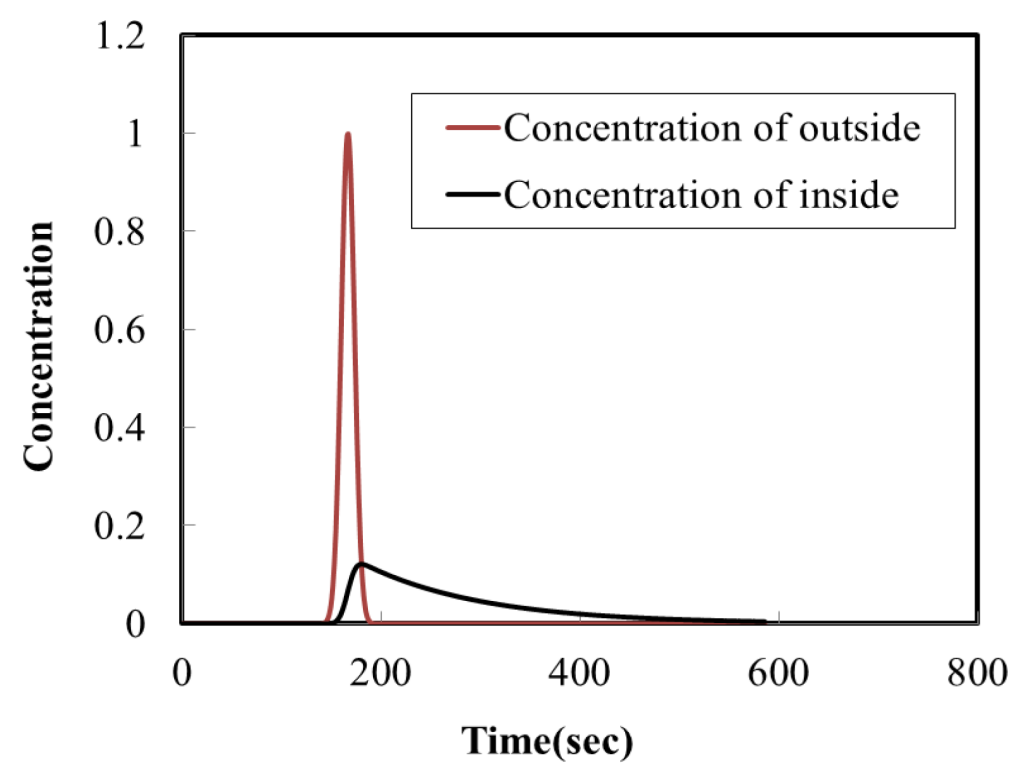

Figure 1: A typical concentration change with time. 


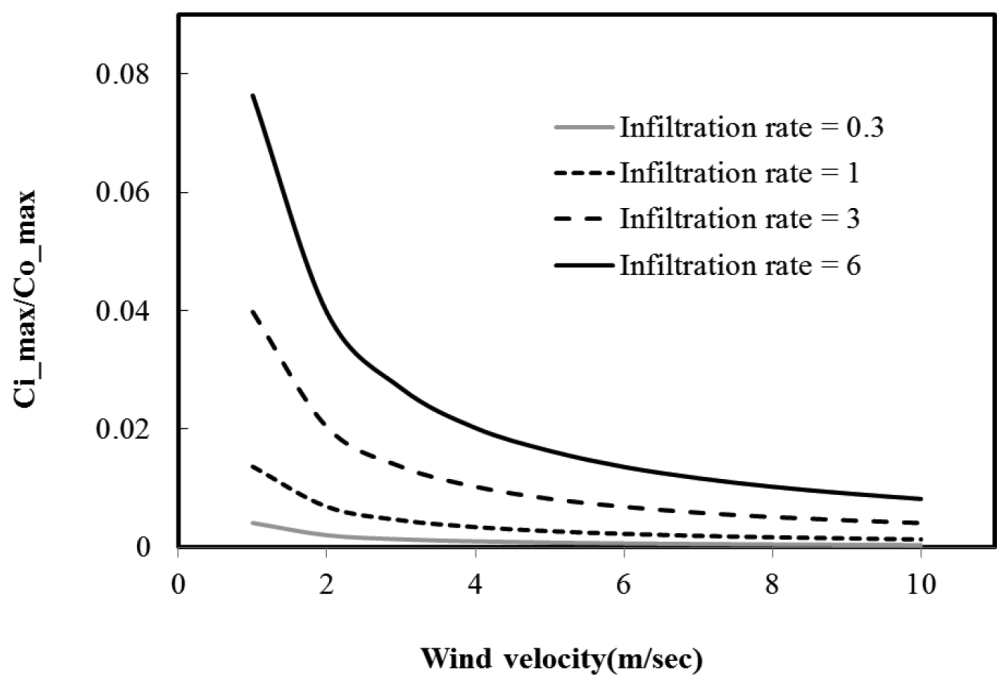

Figure 2: Inside toxic gas concentration with wind velocity and ventilation rate $(\sigma x=20 \mathrm{~m})$.

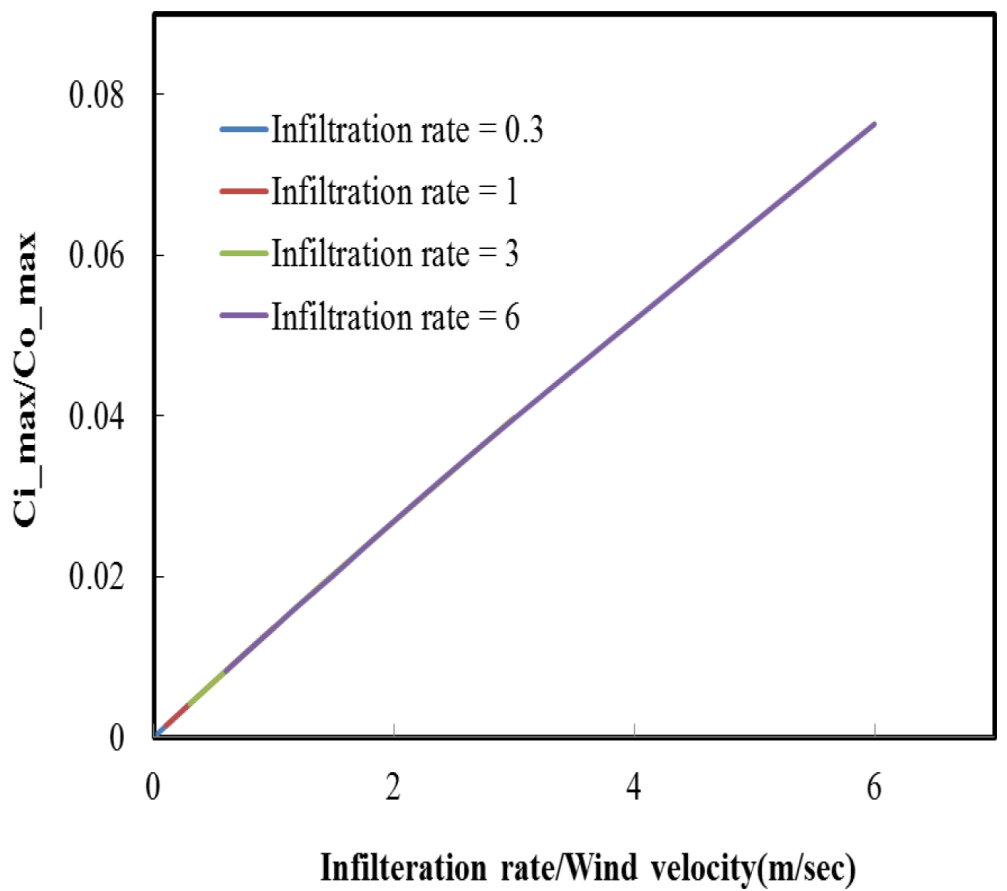

Figure 3: Inside toxic gas concentration with ventilation rate divided by wind velocity $(\sigma \mathrm{x}=20 \mathrm{~m})$.

Substituting the above dispersion coefficients into eqn (3) gives:

$$
C_{0}(x, t)=\frac{50000 Q}{(2 \pi)^{3 / 2} x^{2.39}} \exp \left[-\frac{\left(x-U_{w} t\right)^{2}}{0.0008 x^{1.78}}\right]
$$




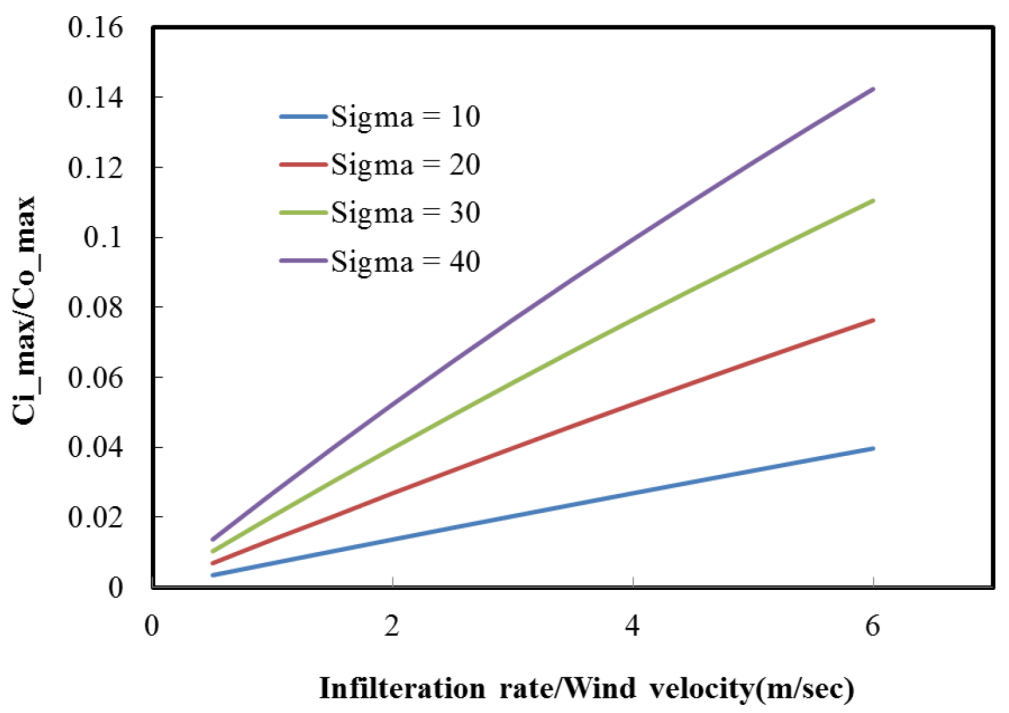

Figure 4: Inside toxic gas concentration with dispersion coefficient, wind velocity, and ventilation

If there is a toxic gas spill from a tank for short time period at a ground level, the concentration of toxic gas at an arbitrary distance from the source in the direction of down-wind can be estimated conservatively by using eqn (12). The maximum concentration decreases with the distance by concentration distribution widening as shown in Fig. 5. The external maximum concentration of toxic gas with the distance of wind direction can be obtained simply from eqn (12) as the following.

$$
C_{0, \max }\left(x, t_{o_{-} \max }\right)=\frac{50000 Q}{(2 \pi)^{3 / 2}} x^{-2.39}
$$

Where $t_{0-\max }$ is the time when the external concentration of toxic gas has a maximum value at a point of down-wind direction, $t_{0 \_ \text {max }}=x / U_{w}$.

The inside maximum concentration of toxic gas in a confined space can be simply estimated by multiplying the external maximum concentration and the ratio of maximum concentrations obtained from Figures 5 or 6 . When we replot the Fig. 5 with the axis of dispersion coefficient, we surprisingly found that the ratio of maximum inside to the external concentration of toxic gas increases linearly with dispersion coefficient as shown in Fig. 6.

$$
C_{i, \max }\left(x, t_{i_{-} \max }\right) / C_{0, \max }\left(x, t_{o_{-} \max }\right)=a_{r} \sigma_{x}
$$

The above equation is valid only for the right term $\left(\mathrm{a}_{r} \sigma_{x}\right)$ of the equation being less than one.

The constants, $\mathrm{a}_{r}$, depend on the ratio of infiltration rate to wind velocity, which are 0.0013 , $0.001,0.0007$, and 0.0003 for the ratio being $2,1.5,1$, and 0.5 , respectively.

$$
a_{r} \cong 0.0007 \frac{\dot{\eta}}{U_{w}}
$$




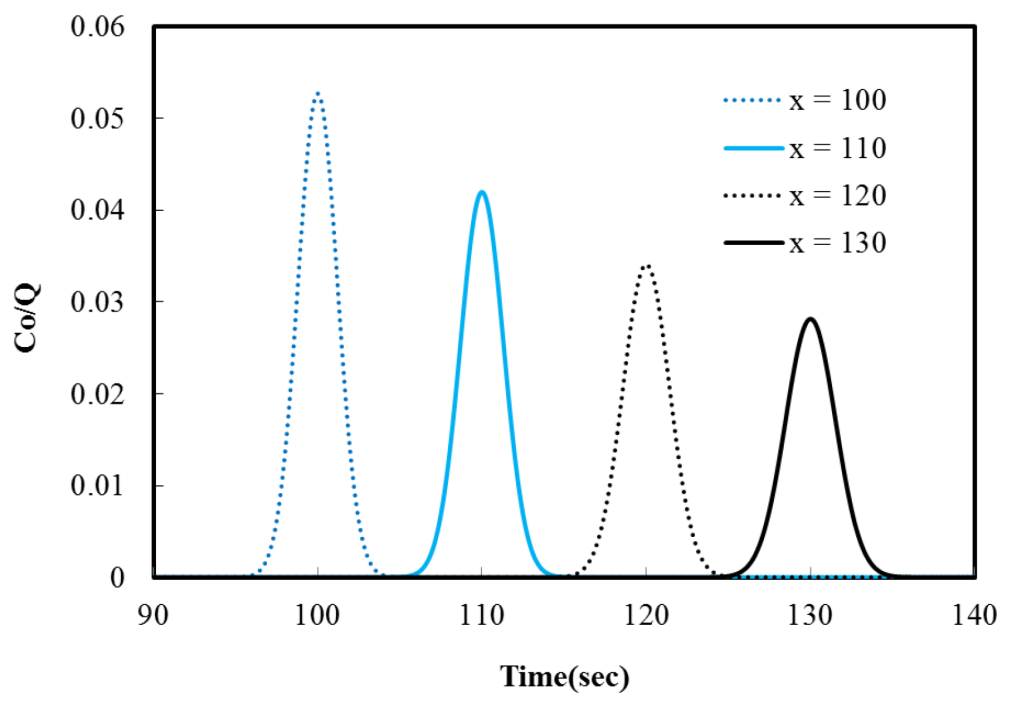

Figure 5: External toxic gas concentration change with time for an accidental release. $\left(U_{w}=1 \mathrm{~m} / \mathrm{sec}\right.$, atmospheric stability class $\left.=\mathrm{E}\right)$.

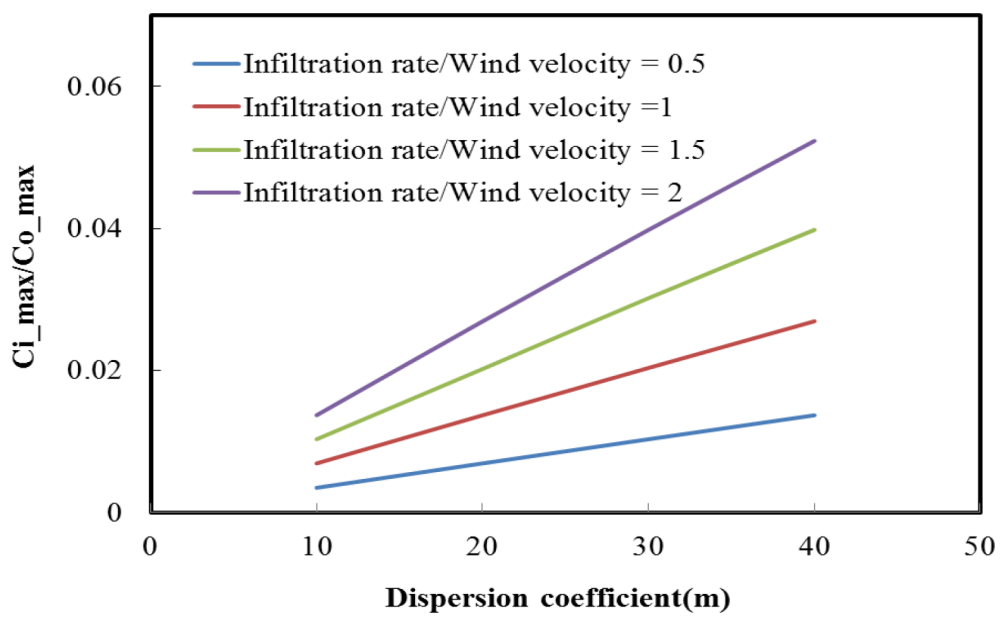

Figure 6: Inside toxic gas concentration with dispersion coefficient.

Removing the maximum external concentration of toxic gas using eqns (13) and (14) the maximum inside concentration is

$$
C_{i, \max }\left(x, t_{i_{-} \max }\right)=\frac{1000 Q}{(2 \pi)^{3 / 2}} a_{r} x^{-1.5}
$$

Where $t_{i-\max }$ is the time when the inside concentration of toxic gas has a maximum value at a point. 
The maximum inside concentration of toxic gas decreases with the distance, but it slowly decreases compared to the maximum external concentration. If the down-wind distance is away from a critical distance, the toxic gas concentration in the building is below lethal concentration for health effects. The external concentration of toxic gas is still higher that the lethal concentration. The critical distance can be estimated by using eqn (16).

$$
x_{c r}=\left[\frac{1000 Q a_{r}}{(2 \pi)^{3 / 2} C_{c r}}\right]^{\frac{1}{1.5}} \cong\left[\frac{0.7 Q}{(2 \pi)^{3 / 2} C_{c r}} \frac{\dot{\eta}}{U_{w}}\right]^{\frac{1}{1.5}}
$$

The critical distance, $\boldsymbol{X}_{\boldsymbol{\alpha}}$, depends on the lethal concentration for a toxic gas, the total amount released gas of the toxic gas, atmospheric stability classes, and the ratio of infiltration rate per wind velocity. If wind velocity increases, arriving time of gas could to a location and the critical distance from the source will be short due to gas could travel with the wind and the constant, $a_{r}$, decrease. Therefore, for puff release and high wind velocity, people leaving the downwind area should be stayed in a building with sealing doors and windows with wet towel and wait until the toxic plume is gone away, because emergency time is not enough to evacuate and the ratio of maximum concentrations is very low.

The distance for no effecting on outdoor person can be estimated by using eqn (13).

$$
x_{n o_{-} e f f e c t} \geq\left[\frac{50000 Q}{(2 \pi)^{3 / 2} C_{c r}}\right]^{\frac{1}{2.39}}
$$

In making decisions to protect people in a hazard area, the decision tree in Fig. 7 can be helpful to make decisions to evacuate, stay at home, and rescue. Adequate time must be allowed for completing the evacuation of people occupying the hazard area. The time depends on the quality of emergency response planning, the characteristics of the area, the available evacuation resources, the density of the population to be evacuated, etc. If the time is enough

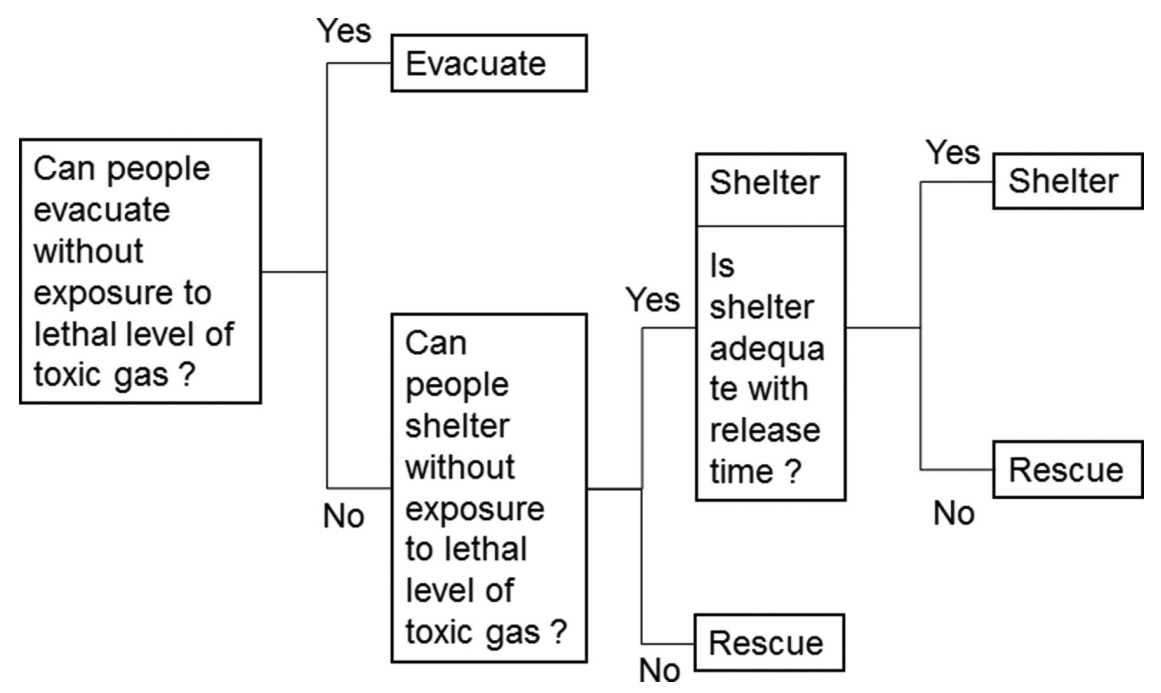

Figure 7: Decision tree to emergency response for a toxic gas release. 
time to evacuate for people in the critical distance before the toxic gas puff arrives, he/she should be evacuated; if not, may need to go inside of the building, close windows and doors until he/she will be rescued. The building within the critical distance has not enough function as a shelter during the toxic gas cloud pass away. People in the range from the critical distance to hazard distance should be stayed in a building with sealing doors and windows and rescued.

\section{CONCLUSIONS}

Toxic gas concentration in a building with toxic gas atmospheric dispersion is very important to help that the local communities are likely to be advised to go indoors and wait until given further advice by the emergency manager. The inside toxic gas concentration of building can be estimated conservatively by using the Gaussian puff model, and simplified equations have been derived to predict the inflicted range to person in the case of a sudden toxic gas release. The inflicted ranges for inside and outside person of building depend on the lethal concentration of a certain toxic gas, the total amount released gas, atmospheric stability classes, and the ratio of infiltration rate per wind velocity. A decision tree is proposed to make decisions to evacuate, stay at home, and rescue by using evacuation time, travelling time of toxic gas, and critical distance which is the inflicted range for a person inside the building. The simplified equations to predict the critical distance and decision tree to emergency response would be helpful in the emergency response plane of toxic gas releases with considering ventilation ratio and meteorological conditions.

\section{REFERENCES}

[1] Lee, K., Kwon, H.M., Cho, S., Kim, J. \& Moon, I., Improvement of safety management system in Korean chemical industry after a large chemical accident. Journal of Loss Prevention in the Process Industries, 42, pp. 6-13, 2015. http://dx.doi.org/10.1016/j.jlp.2015.08.006

[2] Parker, S.T. \& Coffey, C.J., Analytical solutions for exposures and toxic loads in well-mixed shelters in support of shelter-in-place assessments. Journal of Hazardous Materials, 192, pp. 419-422, 2011. http://dx.doi.org/10.1016/j.jhazmat.2011.04.107

[3] Kakko, R., Effects of the ventilation system and chemical reactions on the concentration and individual risk estimates of toxic materials. Journal of Loss Prevention in the Process Industries, 5, pp. 145-152, 1992. http://dx.doi.org/10.1016/0950-4230(92)80017-3

[4] Crowl, D.A. \& Louvar, J.F., Chemical Process Safety Fundamentals with Applications, 3rd edn., Prentice Hall: Upper Saddle River, NJ, pp. 203-204, 2011. 